ANNALS OF “DUNAREA DE JOS” UNIVERSITY OF GALATI

MATHEMATICS, PHYSICS, THEORETICAL MECHANICS

FASCICLE II, YEAR XII (XLIII) 2020, No. 2

DOI: https://doi.org/10.35219/ann-ugal-math-phys-mec.2020.2.16

\title{
Sediments quality assessment in terms of single and integrated indices from Romanian MONITOX network (2019 - 2020)
}

\author{
Liliana Teodorof ${ }^{1, *}$, Adrian Burada ${ }^{1}$, Cristina Despina ${ }^{1}$, Daniela Seceleanu- \\ Odor $^{1}$, Cosmin Spiridon ${ }^{1}$, Mihaela Tiganus ${ }^{1}$, Mihaela-Iuliana Tudor ${ }^{1}$, Marian \\ Tudor $^{1}$, Antoaneta Ene ${ }^{2, *}$, Elena Zubcov ${ }^{2,3}$, Thomas Spanos ${ }^{2,4}$, Oleg Bogdevich ${ }^{2,5}$ \\ ${ }^{1}$ Danube Delta National Institute for Research and Development, Tulcea, Romania, liliana.teodorof@ddni.ro \\ 2 "Dunarea de Jos" University of Galati, INPOLDE research center, ReForm-UDJG Multidisciplinary Platform, \\ Faculty of Sciences and Environment, aene@ugal.ro \\ ${ }^{3}$ Institute of Zoology, Chisinau, Republic of Moldova \\ ${ }^{4}$ International Hellenic University, Kavala, Greece \\ ${ }^{5}$ Institute of Geology and Seismology, Chisinau, Republic of Moldova \\ *Corresponding authors: liliana.teodorof@ddni.ro; aene@ugal.ro
}

\begin{abstract}
The assessment of sediments quality from the Romanian part of MONITOX Network was performed using both single indices - Single Ecological Risk Index $\left(\mathrm{Er}^{\mathrm{Me}}\right)$, Contamination Factor $\left(\mathrm{CF}{ }^{\mathrm{Me}}\right)$, Enrichment Factor $\left(\mathrm{EF}^{\mathrm{Me}}\right)$, Geoaccumulation Index (Igeo ${ }^{\mathrm{Me}}$ ) - and integrated indices - Ecological Risk Index (RI), Global Pollution Index (PLI), Nemerow Pollution Index (PI $\mathrm{N})$, Degree of Contamination $\left(\mathrm{mC}_{\mathrm{d}}\right)$, Probability of Toxicity (MERMQ), Contamination Security Index (CSI) - considering the heavy metals concentrations. The sediments were collected in 2019 and 2020 from 32 sampling points in the system of Danube river - Danube Delta - Black Sea, as follows: Lower Danube RO-BG (1), Lower Danube RO (10), Lower Prut RO-MD border (2), Danube Delta RO-UA border (7), Danube Delta -RO (2), Black-Sea area-RO (10). The selected heavy metals investigated in this work by the ICP-MS technique are: $\mathrm{Cd}, \mathrm{Cr}, \mathrm{Cu}, \mathrm{Pb}, \mathrm{Zn}, \mathrm{Mn}$. For the evaluation of defined indices, the standard quality levels and background concentrations corresponding to the selected heavy metals were used. At the individual and integrated levels, it was observed an annual decreasing trend. The sediments from the Black Sea area are much less contaminated with heavy metals than those from the Lower Danube (Romania), attributed to the historical pollution resulting from anthropogenic activities. CSI - an index that includes the biological effects - shows values indicating that in 2019 and 2020 the sediments were unpolluted with heavy metals; however, the CSI value for both years (0.402 for 2019 and 0.398 for 2020) is very close to 0.5, the limit which separates the two quality classes/risk levels for heavy metals in sediments - uncontaminated and very low contaminated.
\end{abstract}

Keywords: ICP-MS technique, sediment quality, heavy metals, integrated indices, Danube, Black Sea.

\section{INTRODUCTION}

In the last years, all other the world, for the aquatic ecosystems, pollution is an important problem [1]. One major class of pollutants are the heavy metals released in the environment by natural processes or by anthropogenic activities, that affect the water sediments and biota quality, so is very important to evaluate these contaminants.

During the monitoring activities, sediments can be real indicators to evaluate the heavy metals in an aquatic ecosystem [2]. In the system Danube river - Black Sea coast, the sediments act as a real sink for pollutants like heavy metals. This type of contaminants, with a high degree of toxicity and stability against degradation, are accumulated in sediments and bioaccumulated in the trophic chain [2-4]. 
At the river-sea system level, it is very important to understand the risk assessment for heavy metals in sediments, in order to evaluate the specific concentrations and also the possible biological effects [4]. The Danube River collects pollutants, especially heavy metals like cadmium, chromium, copper, lead, and zinc, across its flow through Europe, affecting also the quality of the Black Sea [5].

The main objectives of this research paper are to determine the concentrations of cadmium, chromium, copper, lead, and zinc in 32 bottom sediment samples from the system Danube River Black Sea coast and to assess the ecological risks at the single level and associated with the combined contamination of these heavy metals using different indices [6-18].

All the results from this study were obtained during the monitoring activities of the ENI project with eMS code BSB27 MONITOX (2018-2021), Joint Operational Programme Black Sea Basin 2014-2020 [19].

\section{EXPERIMENTAL}

In the Romanian MONITOX Network area, in the system Danube River - Black Sea coast (Fig.1), in the spring 2019 and 2020, 32 bottom sediments were sampled as follows: Lower Danube RO-BG (1), Lower Danube RO (10), Lower Prut RO-MD border (2), Danube Delta RO-UA border (7), Danube Delta -RO (2), Black-Sea area-RO (10). The samples were dried at room temperature, in the chemistry laboratory of Danube Delta National Institute for Research and Development (DDNI), Tulcea, Romania. According to ISO standard, one gram of each dry sediment was digested in $10 \mathrm{~mL}$ ultrapure nitric acid, using the microwave technique, at microwave oven Anton Paar 3000 and after that, it was diluted with ultrapure water to a $50 \mathrm{~mL}$ volume. The final extract was analysed using the ICP MS Elan DRCe Perkin Elmer mass spectrometry inductively coupled plasma equipment (Figs. 2,3) [20]. The selected heavy metals are cadmium, chromium, copper, lead, and zinc. Using a multielement standard, for each heavy metal, a calibration curve in 5 points was built, with $\mathrm{R}^{2}$ between 0.9995-0.9999. For quality assurance flow charts were performed, using a certified reference material.

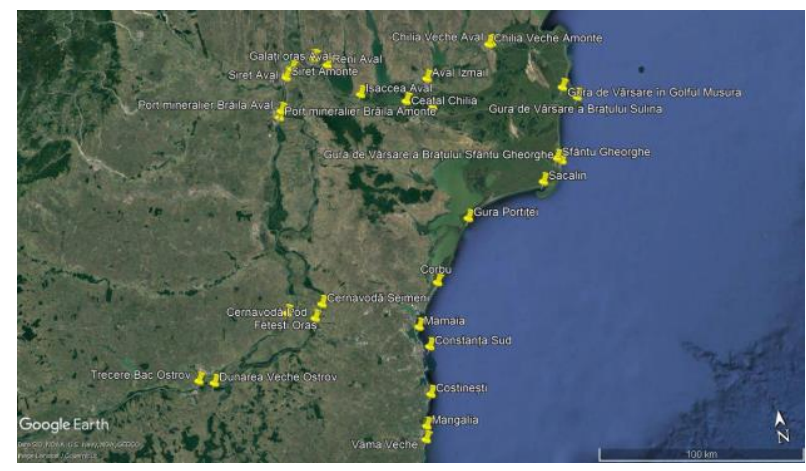

Fig. 1. Romanian MONITOX sampling points

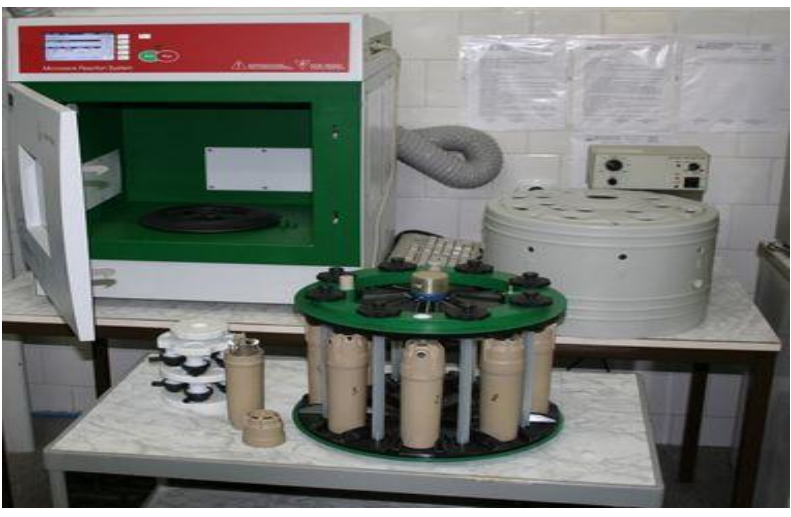

source: DDNI

Fig. 2. Multiwave oven Anton Paar 3000

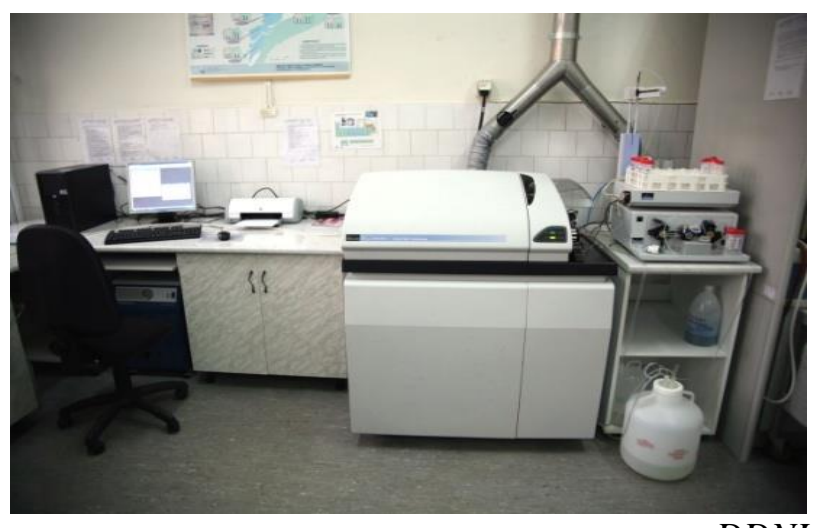

source: DDNI

Fig. 3. ICP-MS Elan DRC-e Perkin Elmer 
In order to assess the sediments' quality in the Romanian MONITOX Network area between the Danube River and Black Sea Coast, it was selected and computed a set of single indices and integrated indices (Tables 1-2), reported to five heavy metals concentrations.

Table 1. Single indices for heavy metals pollution evaluation in sediments

\begin{tabular}{|c|c|c|}
\hline Name & Formula & Explanations \\
\hline $\begin{array}{l}\text { Ecological Risk } \\
\text { Factor } \\
\text { (Hakanson, 1980) } \\
{[6]}\end{array}$ & $\begin{array}{l}\mathrm{C}_{\mathrm{f}}^{\mathrm{Me}}=\mathrm{C}^{\mathrm{Me}} / \mathrm{C} \mathrm{SCM}^{\mathrm{Me}} \\
E_{r}{ }^{M e}=\mathrm{T}_{\mathrm{r}}^{\mathrm{Me}} * \mathrm{C}_{\mathrm{f}}^{\mathrm{Me}}\end{array}$ & $\begin{array}{l}\mathrm{C}^{\mathrm{Me}}=\text { heavy metal concentration } \\
\mathrm{C}_{\mathrm{SCM}}^{\mathrm{Me}}=\text { quality standard for a single heavy metal, according to } \\
\mathrm{Romanian} \text { legislation } \\
\mathrm{T}_{\mathrm{r}}^{\mathrm{Me}}=\text { toxic-response factor for a single heavy metal }(\mathrm{Cd}: 30, \mathrm{Cr}: 2 \text {, } \\
\mathrm{Cu}: 5 . \mathrm{Pb}: 5, \mathrm{Zn:} 1) \\
\mathrm{C}_{\mathrm{f}}^{\mathrm{Me}}=\text { contamination factor for a single heavy metal }\end{array}$ \\
\hline $\begin{array}{l}\text { Enrichment } \\
\text { Factor } \\
\text { (Reiman \& de } \\
\text { Caritat, 2000) [7] }\end{array}$ & $\mathrm{EF}=\left(\mathrm{C}_{\mathrm{i}} / \mathrm{C}_{\mathrm{ie}}\right)_{\mathrm{S}} /\left(\mathrm{C}_{\mathrm{i}} / \mathrm{C}_{\mathrm{ie}}\right)_{\mathrm{RS}}$ & $\begin{array}{l}\mathrm{C}_{\mathrm{is}}==\text { heavy metal concentration from sediment } \\
\text { Cie } \mathrm{s}=\text { imobile heavy metal concentration, from the same sediment } \\
\mathrm{C}_{\mathrm{iRs}}=\text { heavy metal concentration from the sediment considered as } \\
\text { standard sample } \\
\mathrm{C}_{\mathrm{ie} \text { RS }}=\text { imobile heavy metal concentration from the sediment } \\
\text { sample considered as standard sample }\end{array}$ \\
\hline $\begin{array}{l}\text { Geoaccumulation } \\
\text { Index } \\
(\text { Muller, 1969)[8] }\end{array}$ & $\mathrm{I}_{\mathrm{geo}}{ }^{\mathrm{Me}}=\log _{2}\left[\mathrm{C}_{\mathrm{Me}} / 1.5^{*} \mathrm{C}_{\mathrm{Bk}}{ }^{\mathrm{Me}}\right]$ & $\begin{array}{l}\mathrm{C}_{\mathrm{Me}}=\text { heavy metal concentration in the sediment } \\
\mathrm{C}_{\mathrm{Bk}} \mathrm{Me}=\text { background value for the corresponding heavy metal) } \\
\quad(\mathrm{Cd}: 0.25, \mathrm{Cr}: 30, \mathrm{Cu}: 35 . \mathrm{Pb}: 26, \mathrm{Zn}: 130) \\
1.5 \text { =factor used for the possible variations of the background data }\end{array}$ \\
\hline
\end{tabular}

\begin{tabular}{|c|c|c|c|}
\hline \multirow{7}{*}{$\begin{array}{l}\text { Name } \\
\text { Ecological Risk } \\
\text { Factor, Er }\end{array}$} & \multicolumn{3}{|c|}{ Quality class } \\
\hline & Ecological risk class & & $\mathbf{E}_{\mathbf{r}}^{\mathrm{Me}}$ \\
\hline & Low potential ecological risk & & $\mathrm{E}_{\mathrm{r}}^{\mathrm{Me}}<40$ \\
\hline & Moderate potential ecological risk & & $40 \leq \mathrm{E}_{\mathrm{r}} \mathrm{Me}<80$ \\
\hline & Considerable potential ecological risk & & $80 \leq \mathrm{E}_{\mathrm{r}}^{\mathrm{Me}}<160$ \\
\hline & High potential ecological risk & & $160 \leq \mathrm{E}_{\mathrm{r}}^{\mathrm{Me}}<320$ \\
\hline & Very high potential ecological risk & & $\mathrm{E}_{\mathrm{r}}^{\mathrm{Me}} \geq 320$ \\
\hline \multirow[t]{6}{*}{$\begin{array}{l}\text { Enrichment } \\
\text { Factor, EF }\end{array}$} & EF values & & Enrichment class \\
\hline & $\mathrm{EF}<2$ & \multicolumn{2}{|c|}{ Depletion to mineral enrichment } \\
\hline & $2 \leq \mathrm{EF}<5$ & \multicolumn{2}{|c|}{ Moderate enrichment } \\
\hline & $5 \leq \mathrm{EF}<20$ & \multicolumn{2}{|c|}{ Significant enrichment } \\
\hline & $20 \leq \mathrm{EF}<40$ & \multicolumn{2}{|c|}{ Very high enrichment } \\
\hline & $\mathrm{EF} \geq 40$ & \multicolumn{2}{|c|}{ Extremely high enrichment } \\
\hline \multirow{7}{*}{$\begin{array}{l}\text { Geoaccumulation } \\
\text { Index, I geo Me }\end{array}$} & \multicolumn{2}{|l|}{ Sediment quality class } & Values \\
\hline & \multicolumn{2}{|l|}{ Uncontaminated } & $\mathrm{I}_{\text {geo }} \mathrm{Me} \leq 0$ \\
\hline & \multicolumn{2}{|c|}{ Uncontaminated to moderately contaminated } & $0<\mathrm{I}_{\text {geo }} \mathrm{Me}<1$ \\
\hline & \multicolumn{2}{|l|}{ Moderately contaminated } & $1 \leq \mathrm{I}_{\text {geo }} \mathrm{Me}<2$ \\
\hline & \multicolumn{2}{|l|}{ Heavily contaminated } & $2 \leq \mathrm{I}_{\text {geo }} \mathrm{Me}<3$ \\
\hline & \multicolumn{2}{|l|}{ Heavily to extremely contaminated } & $3 \leq \mathrm{I}_{\text {geo }} \mathrm{Me}<4$ \\
\hline & \multicolumn{2}{|l|}{ Extremely contaminated } & $\mathrm{I}_{\text {geo }} \mathrm{Me} \geq 4$ \\
\hline
\end{tabular}


Table 2. Integrated indices for heavy metals pollution evaluation in sediments

\begin{tabular}{|c|c|c|}
\hline Name & Formula & Explanations \\
\hline $\begin{array}{l}\text { Ecological Risk } \\
\text { Index } \\
\text { (Hakanson, 1980) } \\
{[9]}\end{array}$ & $\mathrm{RI}=\sum \mathrm{E}_{\mathrm{r}} \mathrm{Me}$ & $\mathrm{E}_{\mathrm{r}} \mathrm{Me}=$ single ecological risk for the selected heavy metals \\
\hline $\begin{array}{l}\text { Global Pollution } \\
\text { Index } \\
\text { (Liu et al., } \\
2005)[10]\end{array}$ & $\operatorname{PLI}=\left(\prod_{i=1}^{5}\left(\mathrm{C}_{\mathrm{f}} \mathrm{Me}_{\mathrm{i}}\right)\right)^{1 / 5}$ & $\mathrm{E}_{\mathrm{r}} \mathrm{Me}=$ single ecological risk for the selected heavy metals \\
\hline $\begin{array}{l}\text { Nemerow } \\
\text { Pollution Index } \\
\text { (Cheng et al., } \\
\text { 2007)[11] }\end{array}$ & $P I_{N}=\sqrt{\left(\left(\left(C_{f}{ }^{M e} \max \right)^{2}+\left(C_{f}{ }^{M e} \text { average }\right)^{2}\right) / 2\right)}$ & $\begin{array}{l}\mathrm{C}_{\mathrm{f}} \mathrm{Me} \text { max }=\text { maximum contamination factor for all heavy } \\
\text { metals } \\
\mathrm{C}_{\mathrm{f}} \mathrm{Me} \text { average=average of contamination factors for all } \\
\text { heavy metals }\end{array}$ \\
\hline \begin{tabular}{|l|} 
Degree of \\
Contamination \\
(Abrahim \& \\
Parker, 2008 [12]; \\
Kowalska et al., \\
2018 [13]) \\
\end{tabular} & $\mathrm{mC}_{\mathrm{d}}=\underset{\mathrm{i}=1}{\left(\sum_{\mathrm{f}}^{\mathrm{i}}\right) / 5}$ & $\begin{array}{l}\mathrm{C}_{\mathrm{f}}=\mathrm{C}_{\mathrm{Me}} / \mathrm{C}_{\mathrm{Bk}} \\
\mathrm{C}_{\mathrm{f}}{ }^{\mathrm{i}}=\text { contamination factor for heavy metals } \\
\mathrm{C}_{\mathrm{Me}}=\text { heavy metal concentration in sediment } \\
\mathrm{C}_{\mathrm{Bk}}=\text { background concentrations }(\mathrm{Cd}: 0.25, \mathrm{Cr}: 30, \mathrm{Cu}: 35 \\
\mathrm{Pb}: 26, \mathrm{Zn}: 130)\end{array}$ \\
\hline $\begin{array}{l}\text { Probability of } \\
\text { toxicity, mean } \\
\text { ERM quotient } \\
\text { (Gao \& Chen, } \\
\text { 2012)[14] } \\
\end{array}$ & MERMQ $=\sum_{i=1}^{5} \mathrm{C}_{\mathrm{i}} \mathrm{Me}^{\mathrm{E}} \mathrm{ERM}_{\mathrm{i}}$ & $\begin{array}{l}\mathrm{C}_{\mathrm{f}}^{\mathrm{Me}}=\text { heavy metals concentration from sediment } \\
\mathrm{ERM}_{\mathrm{i}}=\text { effect range median values (Long, 1995) [15] } \\
(\mathrm{ERM}, \mathrm{mg} / \mathrm{kg}: \mathrm{Cd}: 9.6, \mathrm{Cr}: 370, \mathrm{Cu}: 270, \mathrm{~Pb}: 218, \mathrm{Zn}: 410)\end{array}$ \\
\hline $\begin{array}{l}\text { Contamination } \\
\text { Security Index } \\
(\text { Pejman } \text { et al., } \\
2015)[2]\end{array}$ & $\mathrm{CSI}=\sum_{\mathrm{i}=1}^{5} \mathrm{~W}_{\mathrm{i}} *\left(\sqrt{ }\left(\mathrm{C}_{\mathrm{i}} \mathrm{Me}^{\mathrm{E}} / \mathrm{ERL}_{\mathrm{i}}\right)+\left(\mathrm{C}_{\mathrm{i}} \mathrm{Me}_{\mathrm{ERM}}\right)^{2}\right)$ & $\begin{array}{l}\mathrm{C}_{\mathrm{f}}^{\mathrm{Me}}=\text { heavy metals concentration from sediment } \\
\mathrm{ERM}_{\mathrm{i}}=\text { effect range median values }(\text { Long, 1995) [15] } \\
\mathrm{ERL}_{\mathrm{i}}=\text { effect range low values (Long, 1995) [15] } \\
\mathrm{w}_{\mathrm{i}}=\text { computed weight of each heavy metal } \\
(\mathrm{ERM}, \mathrm{mg} / \mathrm{kg}: \mathrm{Cd}: 9.6, \mathrm{Cr}: 370, \mathrm{Cu}: 270, \mathrm{~Pb}: 218, \mathrm{Zn}: 410) \\
(\mathrm{ERL}, \mathrm{mg} / \mathrm{kg}: \mathrm{Cd}: 1.2, \mathrm{Cr}: 81, \mathrm{Cu}: 34, \mathrm{~Pb}: 46.7, \mathrm{Zn}: 150)\end{array}$ \\
\hline
\end{tabular}

\begin{tabular}{|c|c|c|c|}
\hline Name & \multicolumn{3}{|r|}{ Quality class } \\
\hline \multirow{5}{*}{$\begin{array}{l}\text { Ecological Risk } \\
\text { Index, RI }\end{array}$} & \multicolumn{2}{|c|}{ Potential ecological risk } & RI values \\
\hline & \multicolumn{2}{|c|}{ Low ecological risk } & $\mathrm{RI}<150$ \\
\hline & \multicolumn{2}{|c|}{ Moderate risk } & $150 \leq \mathrm{RI}<300$ \\
\hline & \multicolumn{2}{|c|}{ Considerable risk } & $300 \leq \mathrm{RI}<600$ \\
\hline & \multicolumn{2}{|c|}{ Very high risk } & $600 \leq \mathrm{RI}$ \\
\hline \multirow{4}{*}{$\begin{array}{l}\text { Global Pollution } \\
\text { Index, PLI }\end{array}$} & PLI values & \multicolumn{2}{|c|}{ Pollution level } \\
\hline & PLI $<1$ & \multicolumn{2}{|c|}{ Not polluted } \\
\hline & PLI = 1 & \multicolumn{2}{|c|}{ Baseline levels of pollution } \\
\hline & PLI $>1$ & \multicolumn{2}{|c|}{ Polluted } \\
\hline \multirow{5}{*}{$\begin{array}{l}\text { Nemerow } \\
\text { Pollution Index, } \\
\text { PI }_{\mathrm{N}}\end{array}$} & \multicolumn{2}{|l|}{$P_{N}$ value } & Pollution level \\
\hline & \multicolumn{2}{|l|}{$\mathrm{PI}_{\mathrm{N}} \leq 1$} & Not polluted \\
\hline & \multicolumn{2}{|l|}{$1<\mathrm{PI} N \leq 2$} & Slightly polluted \\
\hline & \multicolumn{2}{|l|}{$2<\mathrm{PI}_{\mathrm{N}} \leq 3$} & Moderately polluted \\
\hline & \multicolumn{2}{|l|}{$3<\mathrm{PI}_{\mathrm{N}}$} & Severely polluted \\
\hline
\end{tabular}


Table 2. continued

\begin{tabular}{|c|c|c|c|c|}
\hline Name & \multicolumn{4}{|c|}{ Quality class } \\
\hline \multirow{8}{*}{$\begin{array}{l}\text { Degree of } \\
\text { Contamination, } \\
\mathrm{mC}_{\mathrm{d}}\end{array}$} & \multicolumn{2}{|l|}{$\mathrm{mC}_{d}$ value } & \multicolumn{2}{|c|}{ Contamination degree } \\
\hline & \multicolumn{2}{|l|}{$\mathrm{mC}_{\mathrm{d}} \leq 1.5$} & \multicolumn{2}{|c|}{ Nil to very low degree of contamination } \\
\hline & \multicolumn{2}{|l|}{$1.5<\mathrm{mC}_{\mathrm{d}} \leq 2$} & \multicolumn{2}{|c|}{ Low degree of contamination } \\
\hline & \multicolumn{2}{|l|}{$2<\mathrm{mC}_{\mathrm{d}} \leq 4$} & \multicolumn{2}{|c|}{ Moderate degree of contamination } \\
\hline & \multicolumn{2}{|l|}{$4<\mathrm{mC}_{\mathrm{d}} \leq 8$} & \multicolumn{2}{|c|}{ High degree of contamination } \\
\hline & \multicolumn{2}{|l|}{$8<\mathrm{mC}_{\mathrm{d}} \leq 16$} & \multicolumn{2}{|c|}{ Very high degree of contamination } \\
\hline & \multicolumn{2}{|l|}{$16<\mathrm{mC}_{\mathrm{d}} \leq 32$} & \multicolumn{2}{|c|}{ Extremely degree of contamination } \\
\hline & \multicolumn{2}{|l|}{$32<\mathrm{mC}_{\mathrm{d}}$} & \multicolumn{2}{|c|}{ Ultrahigh degree of contamination } \\
\hline \multirow{5}{*}{$\begin{array}{l}\text { Probability of } \\
\text { toxicity, mean } \\
\text { ERM quotient, } \\
\text { MERMQ }\end{array}$} & MERMQ value & \multicolumn{2}{|l|}{ Risk level } & Probability of being toxic \\
\hline & MERMQ $\leq 0.1$ & \multicolumn{2}{|l|}{ Low priority } & $9 \%$ \\
\hline & $0.1<$ MERMQ $\leq 0.5$ & \multicolumn{2}{|c|}{ Medium-low priority } & $21 \%$ \\
\hline & $0.5<$ MERMQ $\leq 1.5$ & \multicolumn{2}{|c|}{ High-medium priority } & $49 \%$ \\
\hline & $1.5<$ MERMQ & \multicolumn{2}{|l|}{ High priority } & $76 \%$ \\
\hline \multirow{10}{*}{$\begin{array}{l}\text { Contamination } \\
\text { Security Index, } \\
\text { CSI }\end{array}$} & \multicolumn{2}{|l|}{ CSI value } & \multicolumn{2}{|c|}{ Risk level } \\
\hline & \multicolumn{2}{|l|}{$\mathrm{CSI}<0.5$} & \multicolumn{2}{|c|}{ Uncontaminated } \\
\hline & \multicolumn{2}{|l|}{$0.5 \leq \mathrm{CSI}<1$} & \multicolumn{2}{|c|}{ Very low contaminated } \\
\hline & \multicolumn{2}{|l|}{$1 \leq \mathrm{CSI}<1.5$} & \multicolumn{2}{|c|}{ Low severity } \\
\hline & \multicolumn{2}{|l|}{$1.5 \leq \mathrm{CSI}<2$} & \multicolumn{2}{|c|}{ Low to moderate severity } \\
\hline & $2 \leq \mathrm{CSI}<2.5$ & & Moderate & \\
\hline & $2.5 \leq \mathrm{CSI}<3$ & & Moderate & verity of contamination \\
\hline & $3 \leq \mathrm{CSI}<4$ & & High seve & tamination \\
\hline & $4 \leq \mathrm{CSI}<5$ & & Very high & f contamination \\
\hline & $5 \leq \mathrm{CSI}$ & & Ultra-high & \\
\hline
\end{tabular}

\section{RESULTS AND DISCUSSION}

In order to assess the sediments' quality in terms of single indices and integrated indices, it was taken into account 5 heavy metals concentrations, in total form, expressed in $\mathrm{mg} / \mathrm{kg}$ of dry substance.

There were computed three single indices and the obtained values were reported to the corresponding quality classes.

Ecological Risk Factor $\left(\mathrm{E}_{\mathrm{r}}^{\mathrm{Me}}\right)$ is quantitatively calculated to express the potential ecological risk with heavy metals, developed by Hakanson $(1980)$ [6,16].

All the selected heavy metals have a low potential ecological risk to sediments $\left(\mathrm{E}_{\mathrm{r}} \mathrm{Me}_{<}<40\right)$. Comparing the selected MONITOX areas, the sediments from the Black-Sea area of Romania have the minimum ecological risk factors, so the heavy metals have the lowest ecological risk to these sediments. The values obtained in 2019 are higher than the values obtained in 2020, probably due to hydrological conditions and the COVID-19 pandemic lockdown. In general, taking into account the values of this indicator, we can establish a general trend, in 2019 and 2020:

$$
\mathrm{E}_{\mathrm{r}}{ }^{\mathrm{Zn}}<\mathrm{E}_{\mathrm{r}}^{\mathrm{Pb}}<\mathrm{E}_{\mathrm{r}} \mathrm{Cu}^{\mathrm{C}}<\mathrm{E}_{\mathrm{r}} \mathrm{Cr}^{\mathrm{C}}<\mathrm{E}_{\mathrm{r}} \mathrm{Cd} \text { (Table 3). }
$$


Table 3. Ecological Risk Factor values determined in sediments from Romanian MONITOX Network Area

\begin{tabular}{|l|c|c|c|c|c|c|c|c|c|c|}
\hline \multirow{2}{*}{ Sampling points } & \multicolumn{2}{|c|}{$\mathbf{E}_{\mathbf{r}}^{\mathbf{C d}}$} & \multicolumn{2}{c|}{$\mathbf{E}_{\mathbf{r}} \mathbf{C r}$} & \multicolumn{2}{c|}{$\mathbf{E}_{\mathbf{r}} \mathbf{C u}$} & \multicolumn{2}{c|}{$\mathbf{E}_{\mathbf{r}}^{\mathbf{P b}}$} & \multicolumn{2}{c|}{$\mathbf{E}_{\mathbf{r}}^{\mathbf{Z n}}$} \\
\cline { 2 - 13 } & $\mathbf{2 0 1 9}$ & $\mathbf{2 0 2 0}$ & $\mathbf{2 0 1 9}$ & $\mathbf{2 0 2 0}$ & $\mathbf{2 0 1 9}$ & $\mathbf{2 0 2 0}$ & $\mathbf{2 0 1 9}$ & $\mathbf{2 0 2 0}$ & $\mathbf{2 0 1 9}$ & $\mathbf{2 0 2 0}$ \\
\hline Lower Danube RO-BG & 2.417 & 2.135 & 0.482 & 0.556 & 0.232 & 0.265 & 0.272 & 0.323 & 0.100 & 0.096 \\
\hline Lower Danube RO & 3.406 & 2.515 & 0.747 & 0.515 & 0.639 & 0.560 & 0.375 & 0.328 & 0.157 & 0.114 \\
\hline $\begin{array}{l}\text { Lower Prut RO-MD } \\
\text { border }\end{array}$ & 5.986 & 3.054 & 0.707 & 0.473 & 1.483 & 0.870 & 0.443 & 0.347 & 0.245 & 0.120 \\
\hline $\begin{array}{l}\text { Danube Delta RO-UA } \\
\text { border }\end{array}$ & 6.082 & 3.492 & 0.650 & 0.442 & 1.205 & 1.048 & 0.422 & 0.412 & 0.223 & 0.131 \\
\hline Danube Delta -RO & 7.359 & 0.611 & 0.764 & 0.299 & 1.829 & 0.114 & 0.530 & 0.112 & 0.284 & 0.028 \\
\hline Black Sea area - RO & 0.618 & 0.352 & 0.241 & 0.073 & 0.389 & 0.045 & 0.168 & 0.053 & 0.068 & 0.001 \\
\hline
\end{tabular}

Enrichment factor $\left(\mathrm{EF}^{\mathrm{Me}}\right)$ is used to show the enrichment degree and metal contamination of an environmental media. EF normalizes the trace element content with respect to a sample reference metal, like manganese [7,17].

In 2019 and 2020, all the sediments are depletion to mineral enrichment with cadmium, copper, and zinc. The sediments from the Lower Danube between Romania and Bulgaria are moderate enrichment with chromium in 2019 and 2020. Significant enrichments have the sediments from the Lower Danube between Romania and Bulgaria with chromium in 2019, lead in 2019 and 2020, Lower Danube of Romania with lead in 2019 and 2020, Lower Prut between Romania and Moldova border with lead in 2020, Danube Delta between Romania and Ukraine border with lead in both years and Danube Delta (Romania) in 2019 with lead. Moderate enrichments have the sediments from Lower Danube RO-BG with chromium, Danube Delta (Romania) with lead in 2020 and Black sea area - RO with lead in 2019 and 2020. A general trend, according to Enrichment Factor values, is:

$$
\mathrm{EF}^{\mathrm{Cu}}<\mathrm{EF}^{\mathrm{Zn}}<\mathrm{EF}^{\mathrm{Cd}}<\mathrm{EF}^{\mathrm{Cr}}<\mathrm{EF}^{\mathrm{Pb}} \text { (Table 4) }
$$

Table 4. Enrichment Factor values determined in Romanian MONITOX Network Area

\begin{tabular}{|l|l|l|l|l|l|l|l|l|l|l|}
\hline \multirow{2}{*}{ Sampling points } & \multicolumn{2}{|c|}{ EF Cd $^{\mathbf{C}}$} & \multicolumn{2}{c|}{ EF Cr } & \multicolumn{2}{c|}{ EF Cu $^{\mathbf{C u}}$ EF Pb } & \multicolumn{2}{c|}{ EF Zn } \\
\cline { 2 - 12 } & $\mathbf{2 0 1 9}$ & $\mathbf{2 0 2 0}$ & $\mathbf{2 0 1 9}$ & $\mathbf{2 0 2 0}$ & $\mathbf{2 0 1 9}$ & $\mathbf{2 0 2 0}$ & $\mathbf{2 0 1 9}$ & $\mathbf{2 0 2 0}$ & $\mathbf{2 0 1 9}$ & $\mathbf{2 0 2 0}$ \\
\hline Lower Danube RO-BG & 1.331 & 1.215 & 2.155 & 2.569 & 0.511 & 0.604 & 7.949 & 9.757 & 1.234 & 1.223 \\
\hline Lower Danube RO & 1.688 & 0.975 & 7.787 & 1.661 & 0.636 & 0.849 & 5.585 & 7.327 & 1.028 & 1.051 \\
\hline Lower Prut RO-MD border & 1.151 & 1.152 & 1.153 & 1.450 & 1.086 & 1.312 & 4.450 & 6.969 & 1.049 & 1.023 \\
\hline $\begin{array}{l}\text { Danube Delta RO-UA } \\
\text { border }\end{array}$ & 1.565 & 1.307 & 1.576 & 1.343 & 1.154 & 1.569 & 6.507 & 8.187 & 1.419 & 1.101 \\
\hline Danube Delta -RO & 1.528 & 0.470 & 1.319 & 1.868 & 1.523 & 0.351 & 5.977 & 4.577 & 1.342 & 0.486 \\
\hline Black Sea area-RO & 0.618 & 0.352 & 0.908 & 0.772 & 0.504 & 0.237 & 3.760 & 3.704 & 0.609 & 0.043 \\
\hline
\end{tabular}

Geoaccumulation Index $\left(\mathrm{Igeo}^{\mathrm{Me}}\right)$ defines metal contamination in sediments by comparing current concentrations with the background concentrations preindustrial levels, developed by Muller (1969) [8]. According to this single indicator, all the sediments are uncontaminated with cadmium, chromium, copper, lead, and zinc. As in the case of the two other indicators, the minimum pollution level is shown by the sediments from the Black-Sea area, Romania. Copper has the minimum geoaccumulation index and chromium the maximum value, and the general trend is:

$$
\mathrm{I}_{\text {geo }} \mathrm{Cu}<\mathrm{I}_{\text {geo }}{ }^{\mathrm{Zn}}<\mathrm{I}_{\text {geo }}{ }^{\mathrm{Pb}}<\mathrm{I}_{\text {geo }}{ }^{\mathrm{Cd}}<\mathrm{I}_{\text {geo }} \mathrm{Cr} \text { (Table 5). }
$$

The single indices establish the quality classes at an individual level for the selected heavy metals. The next level is to integrate all the heavy metals concentrations in different indices to have a general view about sediments quality in the Romanian MONITOX Network Area. There were selected and computed 6 complex integrated indices: Ecological Risk Index, Global Pollution Index, Degree of Contamination, Probability of Toxicity, Contamination Security Index (Table 6). 
Table 5. Geoaccumulation Index values determined in the Romanian MONITOX network area

\begin{tabular}{|l|c|c|c|c|c|c|c|c|c|c|}
\hline \multirow{2}{*}{ Sampling points } & \multicolumn{2}{|c|}{ I geo $_{\text {Cd }}$} & \multicolumn{2}{c|}{ Igeo $_{\text {Cr }}$} & \multicolumn{2}{c|}{ I $_{\text {geo }} \mathbf{C u}$} & \multicolumn{2}{c|}{ I $_{\text {geo }}$ Pb } & \multicolumn{2}{c|}{ Igeo $^{\text {Zn }}$} \\
\cline { 2 - 12 } & $\mathbf{2 0 1 9}$ & $\mathbf{2 0 2 0}$ & $\mathbf{2 0 1 9}$ & $\mathbf{2 0 2 0}$ & $\mathbf{2 0 1 9}$ & $\mathbf{2 0 2 0}$ & $\mathbf{2 0 1 9}$ & $\mathbf{2 0 2 0}$ & $\mathbf{2 0 1 9}$ & $\mathbf{2 0 2 0}$ \\
\hline $\begin{array}{l}\text { Lower Danube RO- } \\
\text { BG }\end{array}$ & -2.540 & -2.720 & -0.900 & -0.694 & -4.822 & -4.628 & -3.021 & -2.773 & -3.707 & -3.767 \\
\hline Lower Danube RO & -2.324 & -2.633 & -0.442 & -0.900 & -4.674 & -3.967 & -3.417 & -2.788 & -3.716 & -3.586 \\
\hline $\begin{array}{l}\text { Lower Prut RO-MD } \\
\text { border }\end{array}$ & -1.265 & -2.206 & -0.352 & -0.929 & -2.248 & -2.919 & -2.368 & -2.668 & -2.452 & -3.433 \\
\hline $\begin{array}{l}\text { Danube Delta RO- } \\
\text { UA border }\end{array}$ & -1.479 & -2.010 & -0.497 & -1.026 & -2.967 & -2.646 & -2.438 & -2.422 & -2.624 & -3.313 \\
\hline Danube Delta -RO & -1.024 & -4.525 & -0.237 & -1.589 & -1.917 & -5.847 & -2.059 & -4.299 & -2.223 & -5.531 \\
\hline $\begin{array}{l}\text { Black Sea area }- \\
\text { RO }\end{array}$ & -4.714 & -5.710 & -3.111 & -3.632 & -6.673 & -7.184 & -5.368 & -5.374 & -5.870 & -9.805 \\
\hline
\end{tabular}

Table 6. Integrated indices values determined in sediments from the Romanian MONITOX network area

\begin{tabular}{|l|c|c|c|c|c|c|c|c|c|c|c|c|}
\hline \multirow{2}{*}{ Sampling points } & \multicolumn{2}{|c|}{ RI } & \multicolumn{2}{c|}{ PLI } & \multicolumn{2}{c|}{ PI } & \multicolumn{2}{c|}{ PI } & \multicolumn{2}{c|}{ mCd } & \multicolumn{2}{c|}{ MERMQ } \\
\cline { 2 - 14 } & $\mathbf{2 0 1 9}$ & $\mathbf{2 0 2 0}$ & $\mathbf{2 0 1 9}$ & $\mathbf{2 0 2 0}$ & $\mathbf{2 0 1 9}$ & $\mathbf{2 0 2 0}$ & $\mathbf{2 0 1 9}$ & $\mathbf{2 0 2 0}$ & $\mathbf{2 0 1 9}$ & $\mathbf{2 0 2 0}$ & $\mathbf{2 0 1 9}$ & $\mathbf{2 0 2 0}$ \\
\hline $\begin{array}{l}\text { Lower Danube } \\
\text { RO-BG }\end{array}$ & 3.503 & 1.493 & 0.188 & 0.199 & 0.241 & 0.278 & 0.252 & 0.289 & 0.281 & 0.307 & 0.027 & 0.030 \\
\hline $\begin{array}{l}\text { Lower Danube } \\
\text { RO }\end{array}$ & 5.324 & 2.350 & 0.292 & 0.232 & 0.381 & 0.258 & 0.401 & 0.274 & 0.555 & 0.200 & 0.043 & 0.022 \\
\hline $\begin{array}{l}\text { Lower Prut RO- } \\
\text { MD border }\end{array}$ & 8.863 & 3.669 & 0.463 & 0.278 & 0.367 & 0.237 & 0.403 & 0.257 & 0.188 & 0.233 & 0.056 & 0.023 \\
\hline $\begin{array}{l}\text { Danube Delta } \\
\text { RO-UA border }\end{array}$ & 8.582 & 3.346 & 0.413 & 0.308 & 0.353 & 0.221 & 0.386 & 0.246 & 0.415 & 0.283 & 0.045 & 0.028 \\
\hline $\begin{array}{l}\text { Danube Delta - } \\
\text { RO }\end{array}$ & 10.767 & 4.266 & 0.543 & 0.073 & 0.428 & 0.150 & 0.471 & 0.154 & 0.403 & 0.362 & 0.065 & 0.038 \\
\hline $\begin{array}{l}\text { Black Sea area }- \\
\text { RO }\end{array}$ & 2.498 & 1.016 & 0.137 & 0.019 & 0.122 & 0.036 & 0.132 & 0.037 & 0.705 & 0.466 & 0.017 & 0.040 \\
\hline
\end{tabular}

According to the Ecological Risk Index determinations, developed by Hakanson (1980) [6], with values in 2019 higher than in 2020, all the sediments from the Romanian MONITOX Network Area have low ecological risk. As a general view, the sediments from Danube Delta - RO have the maximum values of this index, and the sediments from Black Sea Area - RO have the minimum values.

Global Pollution Index, PLI, was calculated as the $5^{\text {th }}$ root of the product of the 5 single indices. The pollution load index (PLI) provides a simple and comparative means for assessing the level of heavy metal pollution [9,10,18]. In 2019 and 2020, all the sediments are unpolluted with heavy metals $(\mathrm{PLI}<1)$, and the minimum values were obtained in the sediments from the Black Sea coast of Romania. The general trend of annual decreasing pollution was highlighted by Gati et al. in 2016 [5]. The value obtained for this indicator was 1.04 for the sediments from Danube Delta [5], higher than 0.308 (2020) and 0.413 (2019).

Nemerow Pollution Index $\left(\mathrm{PI}_{\mathrm{N}}\right)$ was used to assess the total contamination of sediments from the MONITOX network area with heavy metals [11]. According to the values of this complex indicator, all the sediments are not polluted with heavy metals in both years of monitoring in the Romanian MONITOX Network. The minimum pollution has the sediments from the Black Sea coast of Romania and the maximum pollution has the sediments from Danube Delta in 2019.

The Degree of Contamination $(\mathrm{mCd})$ is used for the assessment of the overall degree of contamination with heavy metals in sediments $[12,13]$. From this integrated index point of view, all the sediments present nil to a very low degree of contamination with heavy metals. The sediments from the Black Sea Coast of Romania have the minimum contamination with heavy metals and the sediments from Lower Prut between Romania and Moldova border the maximum contamination in 2019 and 2020. 
The Probability of Toxicity, mean ERM quotient (MERMQ) was used as an instrument to recognize the harmful impact of the heavy metals on sediments $[14,15]$. The risk level of heavy metals to sediments is very low and the probability of the selected heavy metals being toxic is under $9 \%$. The values of MERMQ were obtained, like in other cases, on the Black Sea Coast of Romania.

Contamination Security Index (CSI) is helpful to determine the limit of toxicity of heavy metals, and include also the adverse biological effects [2]. Using the PCA statistics software, Eigenvalues were computed for 2019 (4.166) and 2020 (4.081), values that were necessary to evaluate the CSI Index. Table 10 presents the PC loading values necessary for CSI computing. We evaluated this integrated index in order to establish the quality of all the sediments from the Romanian MONITOX Network. Our results proved that all the sediments in 2019 and 2020 are uncontaminated with heavy metals (Table 11).

Table 10. Loading values for CSI determinations in this work

\begin{tabular}{|l|c|c|c|c|c|}
\hline & Cd & Cr & Cu & Pb & Zn \\
\hline Loading value (PC)-2019 & 0.467 & 0.368 & 0.448 & 0.464 & 0.480 \\
\hline Loading value (PC)-2020 & 0.484 & 0.328 & 0.442 & 0.476 & 0.486 \\
\hline
\end{tabular}

Table 11. Contamination Security Index(CSI) values in 2019 and 2020

\begin{tabular}{|c|c|c|}
\hline Romanian MONITOX network area & $\mathbf{2 0 1 9}$ & $\mathbf{2 0 2 0}$ \\
\hline CSI & 0.402 & 0.398 \\
\hline
\end{tabular}

\section{CONCLUSIONS}

In 2020, the selected heavy metals have a low ecological risk for sediments, with values of Ecological risk factors below the values determined in 2019.

In 2019 and 2020, all the sediments are deficient to minimal enrichment with $\mathrm{Cd}, \mathrm{Cu}, \mathrm{Zn}$. All the sediments have significant enrichment with lead, except the sediments from the Black Sea area (moderate enrichment). In the sediments from the Black Sea area, the trace metal concentration may come entirely from natural weathering processes. The sediment from Lower Danube RO-BG is moderate enrichment with chromium. The sediments from Lower Danube RO have significant enrichment with chromium due to anthropogenic activities.

According to Geoaccumulation Index values, all the sediments are unpolluted with $\mathrm{Cd}, \mathrm{Cr}, \mathrm{Cu}, \mathrm{Pb}$, $\mathrm{Zn}$ in 2019 and 2020.

In 2019 and 2020, at the integrated levels, taking into account the Risk Index, the heavy metals have a low ecological risk for sediments. The values of the Global Pollution Index indicate that the sediments are unpolluted with heavy metals and from Nemerow Pollution Index values, the sediments are not contaminated with heavy metals. Taking into account the new integrated indices, the sediments are nil to a very low degree of contaminations with heavy metals (degree of contaminations values). The probability of heavy metals to be toxic for sediments is $9 \%$, so the risk level is low.

At an individual level, but also at an integrative level, the sediments from the Black Sea area have the minimum contamination with heavy metals and the sediments from the Lower Danube RO area have the maximum contamination, a fact possible attributed to the historical pollution resulting from anthropogenic activities.

Considering the sediments from the Romanian MONITOX Area as a system, we evaluated the Contamination Security Index, CSI, that includes the biological effects, whose values indicated in 2019 and 2020 that the sediments were unpolluted with heavy metals; however, the CSI value for both years is very close to the limit of 0.5 , which separates the two quality classes/risk levels for heavy metals in sediments - uncontaminated and very low contaminated.

Further work is needed at the network level in order to study the spatial-temporal dynamics of heavy metal levels in sediment and correlations with those in water and biota and for other pollutants and microbiological load, recorded after COVID-19 pandemic lockdown and in the pre-lockdown period. 
Acknowledgements: The authors would like to thank the funding of project BSB27-MONITOX (2018-2021), Joint Operational Programme Black Sea Basin 2014-2020.

\section{References}

1. Liu J., Li Y., Zhang B., Cao J., Cao Z., Domagalski J., Ecological risk of heavy metals in sediments of the Luan River source water, Ecotoxicology 18 (2009) 748-758.

2. Pejman A., Bidhendi G.N., Ardestani M., Saeedi M., Baghvand A., A new index for assessing heavy metals contamination in sediments: A case study, Ecological Indicators, 58 (2015) 365-373.

3. Diagomanolin V., Farhang M., Ghazi-Khansari M., Jafarzadeh N., Heavy metals (Ni, Cr, Cu) in the Karoon waterway river, Iran, Toxicology Letter 151 (2004) 63-68.

4. Zhang W., Liu X., Cheng H., Zeng E.Y., Hu Y., Heavy metal pollution in sediments of a typical mariculture zone in South China, Marine Pollution Bulletin 64 (2012) 712-720.

5. Gati G., Pop C, Brudaşcă F., Gurzău A.E., Spînu M., The ecological risk of heavy metals in sediment from the Danube Delta, Ecotoxicology 25 (4) (2016) 688-696.

6. Hắkanson L., An ecological risk index for aquatic pollution control: a sedimentological approach, Water Research 14 (1980) 975-1001.

7. Reimann C., de Caritat P., Intrinsic flaws of element enrichment factors (EFs) in environmental geochemistry, Environmental Science and Technology 34 (2000) 5084-5091.

8. Muller G., Index of geoaccumulation in sediments of the Rhine River, Geology Journal 2 (1969) 108-118.

9. Tomlison L., Wilson G., Harris R., Jeffrey D.W., Problems in the assessments of heavy-metal levels in estuaries and formation of a pollution index, Helgol Meeresunters 33 (1980) $566-575$.

10. Liu W.H., Zhao J.Z., Ouyang Z.Y., Söderlund L., Liu G.H., Impacts of sewage irrigation on heavy metal distribution and contamination in Beijing, China, Environment International 31(6) (2005) 805-812.

11. Cheng J. L., Shi Z., Zhu Y. W., Assessment and Mapping of Environmental Quality in Agricultural Soils of Zhejiang Province, China, Journal of Environmental Sciences 19 (2007) 5054.

12. Abrahim G. M. S., Parker, R. J., Assessment of heavy metal enrichment factors and the degree of contamination in marine sediments from Tamaki Estuary, Auckland, New Zealand, Environmental Monitoring and Assessment 136 (2008) 227-238.

13. Kowalska J.B., Mazurek R., Gąsiorek M., Zaleski T., Pollution indices as useful tools for the comprehensive evaluation of the degree of soil contamination-A review, Environmental Geochemistry and Health 40 (2018) 2395-2420.

14. Gao X., Chen C.T., Heavy metal pollution status in surface sediments of the coastal Bohai Bay, Water Research 46(6) (2012) 1901-1911.

15. Long E.R., Ranges in chemical concentrations in sediments associated with adverse biological effects, Marine Pollution Bulletin 24 (1) (1995) 38-45.

16. Gong Q., Deng J., Yunchuan X., Qingfei W., Yang L., Calculating pollution indices by heavy metals in ecological geochemistry, assesment and a case study in parks of Beijing, Journal of China University of Geosciences 19 (3) (2008) 230-241.

17. Sutherland R.A., Bed sediment-associated trace metals in an urban stream, Oahu, Hawaii, Environmental Geology 39(6) (2000) 611-627.

18. Cabrera F., Clemente L., Diaz Barrientos E., Lopez R., Murillo J.M., Heavy metal pollution of soils affected by the Guadiamar toxic flood, Science Total Environment 242 (1999) 117 - 129.

19. Ene A., Zubcov E., Spanos T., Bogdevich O., Teodorof L., Interdisciplinary Cooperation for Ecological Monitoring in the Black Sea Basin, Abstract book MONITOX International Symposium "Deltas and Wetlands", September 15-17, 2019, Tulcea, Romania, C.I.T.D.D, 2019, pp.17-18, 2019.

20. Despina C., Teodorof L., Burada A., Seceleanu-Odor D., Tiganus M., Tudor I.-M., Ibram O., Spiridon C., Nastase A., Tiganov G., Ene A., Danube Delta Biosphere Reserve - ten years of toxic substances monitoring, Annals Dunarea de Jos Univ. Galati, Fasc. II. Mathematics, Physics, Theoretical Mechanics, 40(1) (2017) 55-60. 